\title{
COMMUTATORS OF SINGULAR INTEGRALS REVISITED
}

\author{
ANDREI K. LERNER, SHELDY OMBROSI, AND ISRAEL P. RIVERA-RÍOS
}

\begin{abstract}
We obtain a Bloom-type characterization of the two-weighted boundedness of iterated commutators of singular integrals. The necessity is established for a rather wide class of operators, providing a new result even in the unweighted setting for the first order commutators.
\end{abstract}

\section{INTRODUCTION}

Given a linear operator $T$ and a locally integrable function $b$, define the commutator $[b, T]$ of $T$ and $b$ by

$$
[b, T] f(x)=b(x) T(f)(x)-T(b f)(x) .
$$

The iterated commutators $T_{b}^{m}, m \in \mathbb{N}$, are defined inductively by

$$
T_{b}^{m} f=\left[b, T_{b}^{m-1}\right] f, \quad T_{b}^{1} f=[b, T] f .
$$

We say that a linear operator $T$ is an $\omega$-Calderón-Zygmund operator on $\mathbb{R}^{n}$ if $T$ is $L^{2}$ bounded, and can be represented as

$$
T f(x)=\int_{\mathbb{R}^{n}} K(x, y) f(y) d y \quad \text { for all } x \notin \operatorname{supp} f,
$$

with kernel $K$ satisfying the size condition $|K(x, y)| \leq \frac{C_{K}}{|x-y|^{n}}, x \neq y$, and the smoothness condition

$$
\left|K(x, y)-K\left(x^{\prime}, y\right)\right|+\left|K(y, x)-K\left(y, x^{\prime}\right)\right| \leq \omega\left(\frac{\left|x-x^{\prime}\right|}{|x-y|}\right) \frac{1}{|x-y|^{n}}
$$

for $|x-y|>2\left|x-x^{\prime}\right|$, where $\omega:[0,1] \rightarrow[0, \infty)$ is continuous, increasing, subadditive and $\omega(0)=0$. We say that $\omega$ satisfies the Dini condition if $\int_{0}^{1} \omega(t) \frac{d t}{t}<\infty$.

In this paper, we will prove the following result.

Theorem 1.1. Let $\mu, \lambda \in A_{p}, 1<p<\infty$. Further, let $\nu=\left(\frac{\mu}{\lambda}\right)^{\frac{1}{p}}$ and $m \in \mathbb{N}$.

(i) If $b \in B M O_{\nu^{1 / m}}$, then for every $\omega$-Calderón-Zygmund operator $T$ on $\mathbb{R}^{n}$ with $\omega$ satisfying the Dini condition,

$$
\left\|T_{b}^{m} f\right\|_{L^{p}(\lambda)} \leq c_{n, m, T}\|b\|_{B M O_{\nu^{1 / m}}}^{m}\left([\lambda]_{A_{p}}[\mu]_{A_{p}}\right)^{\frac{m+1}{2} \max \left\{1, \frac{1}{p-1}\right\}}\|f\|_{L^{p}(\mu)} .
$$

(ii) Let $T_{\Omega}$ be an operator defined by 1.1) with $K(x, y)=\Omega\left(\frac{x-y}{|x-y|}\right) \frac{1}{|x-y|^{n}}$, where $\Omega$ is a measurable function on $S^{n-1}$, which does not change sign and is not equivalent to zero on some open subset from $S^{n-1}$. If there is $c>0$ such that for every bounded measurable set $E \subset \mathbb{R}^{n}$,

$$
\left\|\left(T_{\Omega}\right)_{b}^{m}\left(\chi_{E}\right)\right\|_{L^{p}(\lambda)} \leq c \mu(E)^{1 / p}
$$

then $b \in B M O_{\nu^{1 / m}}$.

2010 Mathematics Subject Classification. 42B20, 42B25.

Key words and phrases. Commutators, weighted inequalities, Calderón-Zygmund operators. 
Remark 1.2. We emphasize that in part (ii) of Theorem 1.1, no size and regularity assumptions on $\Omega$ are imposed. It will be useful, however, to distinguish a class of operators satisfying both parts of the theorem. Assume that

$$
T_{\Omega} f(x)=\mathrm{p} . \mathrm{v} \cdot \int_{\mathbb{R}^{n}} f(x-y) \frac{\Omega(y /|y|)}{|y|^{n}} d y,
$$

where $\Omega$ is continuous on $S^{n-1}$, not identically zero and $\int_{S^{n-1}} \Omega d \sigma=0$. Assuming additionally that

$$
\omega(\delta)=\sup _{\left|\theta-\theta^{\prime}\right| \leq \delta}\left|\Omega(\theta)-\Omega\left(\theta^{\prime}\right)\right|
$$

satisfies the Dini condition, we obtain that $T_{\Omega}$ satisfies both parts of Theorem 1.1 .

Recall that $b \in B M O_{\eta}$ (for a given weight $\eta$ ) if

$$
\|b\|_{B M O_{\eta}}=\sup _{Q} \frac{1}{\eta(Q)} \int_{Q}\left|b(x)-b_{Q}\right| d x<\infty,
$$

where the supremum is taken over all cubes $Q \subset \mathbb{R}^{n}$. Here we use the standard notations $\eta(Q)=\int_{Q} \eta$ and $b_{Q}=\frac{1}{|Q|} \int_{Q} b$. We also recall that $w \in A_{p}, 1<p<\infty$, if

$$
[w]_{A_{p}}=\sup _{Q \subset \mathbb{R}^{n}}\left(\frac{1}{|Q|} \int_{Q} w\right)\left(\frac{1}{|Q|} \int_{Q} w^{-\frac{1}{p-1}}\right)^{p-1}<\infty .
$$

In what follows, we present a brief history preceding Theorem 1.1, and, in parallel, we outline our novel points.

- Assume first that $m=1$ and $\lambda=\mu \equiv 1$. In this case Theorem 1.1 was obtained in the celebrated work by Coifman, Rochberg and Weiss [4].

The necessity of $B M O$, expressed in part (ii), was obtained in [4] under the assumption that $\left[b, R_{j}\right]$ is bounded on $L^{p}$ for every Riesz transform $R_{j}$. Later this assumption was relaxed in the works by Janson [16] and Uchiyama [21]. It was shown there that the boundedness of $\left[b, T_{\Omega}\right]$ on $L^{p}$ (where $T_{\Omega}$ is defined as in Remark 1.2 with $\Omega \in C^{\infty}\left(S^{n-1}\right)$ in [16] and $\Omega$ is Lipschitz continuous in [21]) implies $b \in B M O$.

Our novel points in part (ii) (even in the unweighted case and when $m=1$ ) are a much wider class of operators (which includes, for instance, a class of rough singular integrals) and the fact that the restricted strong type $(p, p)$ of $\left[b, T_{\Omega}\right]$ (instead of the usual strong type $(p, p)$ in $[4,16,21])$ implies $b \in B M O$.

- Assume that $m=1$ and $\lambda, \mu \in A_{p}$. In the one-dimensional case this result was obtained by Bloom [2]. Recently it was extended to higher dimensions by Holmes, Lacey and Wick [10]. Later, a quantitative form of this statement, expressed in estimate (1.2), was obtained by the authors in [19].

As in the unweighted case, part (ii) is new in such generality. In [10] this part was obtained, similarly to [4], assuming that $\left[b, R_{j}\right]$ is bounded from $L^{p}(\mu)$ to $L^{p}(\lambda)$ for every Riesz transform $R_{j}$.

- Assume that $m \geq 2$. Suppose now that $\lambda, \mu \in A_{p}$. In the early 90s, García-Cuerva, Harboure, Segovia and Torrea [7] proved for a class of strongly singular integrals $S$ that $b \in B M O_{\nu^{1 / m}}$ implies $S_{b}^{m}: L^{p}(\mu) \rightarrow L^{p}(\lambda)$. It was pointed out in [7] that similar methods can be used to obtain the corresponding estimates for Calderón-Zygmund operators.

Estimate (1.2) represents a quantitative version of that statement. It looks like a natural extension of the case $m=1$ obtained in [19]. Notice, however, that it does not seem that this estimate can be deduced via a simple inductive argument. Observe also that in the case of equal weights, 1.2 recovers the sharp dependence on the $A_{p}$ constant established 
by Chung, Pereyra and Pérez $\left[3\right.$ for every $m \geq 1$. This indicates that the exponent $\frac{m+1}{2}$ in (1.2) cannot be improved.

Recently, Holmes and Wick [11] obtained the $L^{p}(\mu) \rightarrow L^{p}(\lambda)$ boundedness of $T_{b}^{m}$ under the different assumption $b \in B M O \cap B M O_{\nu}$ with $\nu=\left(\frac{\mu}{\lambda}\right)^{\frac{1}{p}}$. Hytönen $\mid 14$ provided a simpler argument for this result based on the conjugation method. We will show below (see Remark 4.7 in Section 4) that the assumption $b \in B M O_{\nu^{1 / m}}$ is less restrictive than $b \in B M O \cap B M O_{\nu}$.

Part (ii) of Theorem 1.1 for $m \geq 2$ is new even for the commutators of the Hilbert transform. Notice that in [7] the necessity of $b \in B M O_{\nu^{1 / m}}$ was deduced from the $L^{p}(\mu) \rightarrow$ $L^{p}(\lambda)$ boundedness of the commutators of the Hardy-Littlewood maximal operator.

Summarizing, our new contribution in Theorem 1.1 is the following.

- If $m \geq 2$, then both parts of Theorem 1.1 are new.

- If $m=1$, then part (ii) provides a much wider class of operators comparing to the previous works, both in weighted and unweighted cases.

- In part (ii), the necessity of $B M O_{\nu^{1 / m}}$ follows from the weighted restricted strong type $(p, p)$ estimates.

The rest of the paper is organized as follows. Section 2 is devoted to present some needed preliminary results. In Section 3 we prove Theorem 1.1. The last section contains some further comments and remarks related to Theorem 1.1.

\section{PRELiminaries}

2.1. $A_{\infty}$ weights. Define the $A_{\infty}$ class of weights by $A_{\infty}=\cup_{p>1} A_{p}$. We mention several well known properties of $A_{\infty}$ weights (see, e.g., [8, Ch. 9]). First, if $w \in A_{\infty}$, then $w$ is doubling, that is, for every $\lambda>1$, there is $c>0$ such that for all cubes $Q$,

$$
w(\lambda Q) \leq c w(Q)
$$

where $\lambda Q$ denotes the cube with the same center as $Q$ and side length $\lambda$ times that of $Q$. Second, for every $0<\alpha<1$, there exists $0<\beta<1$ such that for every cube $Q$ and every measurable set $E \subset Q$ with $|E| \geq \alpha|Q|$ one has

$$
w(E) \geq \beta w(Q) .
$$

Next, there exists $\gamma>0$ such that for every cube $Q$,

$$
\left|\left\{x \in Q: w(x) \geq \gamma w_{Q}\right\}\right| \geq \frac{1}{2}|Q| .
$$

In particular, this property implies immediately that for every cube $Q$ and for all $0<\delta<1$,

$$
\frac{1}{|Q|} \int_{Q} w \leq \frac{2^{1 / \delta}}{\gamma}\left(\frac{1}{|Q|} \int_{Q} w^{\delta}\right)^{1 / \delta}
$$

2.2. Sparse families and mean oscillations. Given a cube $Q_{0} \subset \mathbb{R}^{n}$, let $\mathcal{D}\left(Q_{0}\right)$ denote the set of all dyadic cubes with respect to $Q_{0}$, that is, the cubes obtained by repeated subdivision of $Q_{0}$ and each of its descendants into $2^{n}$ congruent subcubes.

A dyadic lattice $\mathscr{D}$ in $\mathbb{R}^{n}$ is any collection of cubes such that

(i) if $Q \in \mathscr{D}$, then each child of $Q$ is in $\mathscr{D}$ as well;

(ii) every 2 cubes $Q^{\prime}, Q^{\prime \prime} \in \mathscr{D}$ have a common ancestor, i.e., there exists $Q \in \mathscr{D}$ such that $Q^{\prime}, Q^{\prime \prime} \in \mathcal{D}(Q)$

(iii) for every compact set $K \subset \mathbb{R}^{n}$, there exists a cube $Q \in \mathscr{D}$ containing $K$. 
A family of cubes $\mathcal{S}$ is called sparse if there exists $0<\alpha<1$ such that for every $Q \in \mathcal{S}$ one can find a measurable set $E_{Q} \subset Q$ with $\left|E_{Q}\right| \geq \alpha|Q|$, and the sets $\left\{E_{Q}\right\}_{Q \in \mathcal{S}}$ are pairwise disjoint.

Given a measurable function $f$ on $\mathbb{R}^{n}$ and a cube $Q$, the local mean oscillation of $f$ on $Q$ is defined by

$$
\omega_{\lambda}(f ; Q)=\inf _{c \in \mathbb{R}}\left((f-c) \chi_{Q}\right)^{*}(\lambda|Q|) \quad(0<\lambda<1),
$$

where $f^{*}$ denotes the non-increasing rearrangement of $f$.

By a median value of $f$ over a measurable set $E$ of positive finite measure we mean a possibly nonunique, real number $m_{f}(E)$ such that

$$
\max \left(\left|\left\{x \in E: f(x)>m_{f}(E)\right\}\right|,\left|\left\{x \in E: f(x)<m_{f}(E)\right\}\right|\right) \leq|E| / 2 .
$$

Notice that, by Chebyshev's inequality,

$$
\sup _{Q} \omega_{\lambda}(f ; Q) \leq \frac{1}{\lambda}\|f\|_{B M O} \quad(0<\lambda<1) .
$$

By a well known result due to John [17] and Strömberg [20], the converse estimate holds as well for $\lambda \leq \frac{1}{2}$, thus providing an alternative characterization of $B M O$ in terms of local mean oscillations.

Similarly to 2.4, for every weight $\eta$,

$$
\sup _{Q} \omega_{\lambda}(f ; Q) \frac{|Q|}{\eta(Q)} \leq \frac{1}{\lambda}\|f\|_{B M O_{\eta}} \quad(0<\lambda<1) .
$$

We will show that assuming $\eta \in A_{\infty}$, the full analogue of the John-Strömberg result holds for $\lambda \leq \lambda_{n}$. This fact is a simple application of the following result due to the first author [18] and stated below in the refined form obtained by Hytönen [13]: for every measurable function $f$ on a cube $Q$, there exists a (possibly empty) $\frac{1}{2}$-sparse family $\mathcal{S}$ of cubes from $\mathcal{D}(Q)$ such that for a.e. $x \in Q$,

$$
\left|f(x)-m_{f}(Q)\right| \leq 2 \sum_{P \in \mathcal{S}} \omega_{\frac{1}{2^{n+2}}}(f ; P) \chi_{P}(x) .
$$

Lemma 2.1. Let $\eta \in A_{\infty}$. Then

$$
\|f\|_{B M O_{\eta}} \leq \sup _{Q} \omega_{\lambda}(f ; Q) \frac{|Q|}{\eta(Q)} \quad\left(0<\lambda \leq \frac{1}{2^{n+2}}\right)
$$

where $c$ depends only on $\eta$.

Proof. Since $\omega_{\lambda}(f ; Q)$ is non-increasing in $\lambda$, it sufficed to prove 2.6$)$ for $\lambda=\frac{1}{2^{n+2}}$. Let $Q$ be an arbitrary cube. Then, by (2.5),

$$
\begin{aligned}
\int_{Q}\left|f-f_{Q}\right| d x & \leq 2 \int_{Q}\left|f-m_{f}(Q)\right| d x \leq 4 \sum_{P \in \mathcal{S}, P \subseteq Q} \omega_{\frac{1}{2^{n+2}}}(f ; P)|P| \\
& \leq 4\left(\sup _{P} \omega_{\frac{1}{2^{n+2}}}(f ; P) \frac{|P|}{\eta(P)}\right) \sum_{P \in \mathcal{S}, P \subseteq Q} \eta(P) .
\end{aligned}
$$

Using that $\mathcal{S}$ is sparse and applying $(2.2)$, we obtain

$$
\sum_{P \in \mathcal{S}, P \subseteq Q} \eta(P) \leq c \sum_{P \in \mathcal{S}, P \subseteq Q} \eta\left(E_{P}\right) \leq c \eta(Q),
$$

which, along with the previous estimate, completes the proof. 
We will also use the following result proved recently in 19 , Lemma 5.1] and closely related to (2.5): given a dyadic lattice $\mathscr{D}$ and a sparse family $\mathcal{S} \subset \mathscr{D}$, there exists a sparse family $\tilde{\mathcal{S}} \subset \mathscr{D}$ containing $\mathcal{S}$ and such that if $Q \in \tilde{\mathcal{S}}$, then for a.e. $x \in Q$,

$$
\left|f(x)-f_{Q}\right| \leq 2^{n+2} \sum_{P \in \tilde{\mathcal{S}}, P \subseteq Q}\left(\frac{1}{|P|} \int_{P}\left|f-f_{P}\right|\right) \chi_{P}(x) .
$$

\section{Proof of Theorem 1.1}

3.1. Proof of part (i). We recall that in that in the case $m=1$, the proof provided in 19 relied upon the sparse domination result

$$
\begin{aligned}
|[b, T] f(x)| & \leq c_{n, T} \sum_{j=1}^{3^{n}} \sum_{Q \in \mathcal{S}_{j}}\left|b(x)-b_{Q}\right|\left(\frac{1}{|Q|} \int_{Q}|f|\right) \chi_{Q}(x)+ \\
& +c_{n, T} \sum_{j=1}^{3^{n}} \sum_{Q \in \mathcal{S}_{j}}\left(\frac{1}{|Q|} \int_{Q}\left|b-b_{Q}\right||f|\right) \chi_{Q}(x),
\end{aligned}
$$

combined with (2.7) and the sharp weighted $L^{p}$ bound for sparse operators.

To settle the case $m \geq 2$, we hinge upon the counterpart for iterated commutators obtained in 15$]$,

$$
\left|T_{b}^{m} f(x)\right| \leq c_{n, T} \sum_{j=1}^{3^{n}} \sum_{k=0}^{m}\left(\begin{array}{c}
m \\
k
\end{array}\right) \sum_{Q \in \mathcal{S}_{j}}\left|b(x)-b_{Q}\right|^{m-k}\left(\frac{1}{|Q|} \int_{Q}\left|b-b_{Q}\right|^{k}|f|\right) \chi_{Q}(x) .
$$

The idea of the proof consists in combining that sparse domination with (2.7). However, in contrast with the case $m=1$, the sparse terms that arise after applying (2.7) may be rised to a power larger than 1. We will deal with those terms exploiting the dyadic structure of the sparse family via (3.5). Bearing those ideas in mind, the rest of the proof can be carried out taking into account the selfadjointness of sparse operators and the sharp weighted $L^{p}$ bound that they satisfy.

We begin our argument as follows. Observe that taking into account (3.2) it suffices to provide suitable estimates for

$$
A_{b}^{m, k} f(x)=\sum_{Q \in \mathcal{S}}\left|b(x)-b_{Q}\right|^{m-k}\left(\frac{1}{|Q|} \int_{Q}\left|b-b_{Q}\right|^{k}|f|\right) \chi_{Q}(x),
$$

where $\mathcal{S}$ is a sparse family from some dyadic lattice $\mathscr{D}$.

We start observing that, by duality,

$$
\left\|A_{b}^{m, k} f\right\|_{L^{p}(\lambda)} \leq \sup _{\|g\|_{L^{p^{\prime}}(\lambda)}} \sum_{Q \in \mathcal{S}}\left(\int_{Q}|g \lambda|\left|b-b_{Q}\right|^{m-k}\right) \frac{1}{|Q|} \int_{Q}\left|b-b_{Q}\right|^{k}|f| .
$$

By 2.7), there exists a sparse family $\tilde{\mathcal{S}} \subset \mathscr{D}$ containing $\mathcal{S}$ and such that if $Q \in \tilde{\mathcal{S}}$, then for a.e. $x \in Q$,

$$
\left|b(x)-b_{Q}\right| \leq 2^{n+2} \sum_{P \in \tilde{\mathcal{S}}, P \subseteq Q}\left(\frac{1}{|P|} \int_{P}\left|b-b_{P}\right|\right) \chi_{P}(x) .
$$

From this, assuming that $b \in B M O_{\eta}$, where $\eta$ is a weight to be chosen later, we obtain

$$
\left|b(x)-b_{Q}\right| \leq 2^{n+2}\|b\|_{B M O_{\eta}} \sum_{P \in \tilde{\mathcal{S}}, P \subseteq Q} \eta_{P} \chi_{P}(x) .
$$


Hence,

$$
\begin{aligned}
& \sum_{Q \in \mathcal{S}}\left(\int_{Q}|g \lambda|\left|b-b_{Q}\right|^{m-k}\right) \frac{1}{|Q|} \int_{Q}\left|b-b_{Q}\right|^{k}|f| \\
& \leq c\|b\|_{B M O_{\eta}}^{m} \sum_{Q \in \tilde{\mathcal{S}}}\left(\frac{1}{|Q|} \int_{Q}|g \lambda|\left(\sum_{P \in \tilde{\mathcal{S}}, P \subseteq Q} \eta_{P} \chi_{P}\right)^{m-k}\right) \\
& \times\left(\frac{1}{|Q|} \int_{Q}\left(\sum_{P \in \tilde{\mathcal{S}}, P \subseteq Q} \eta_{P} \chi_{P}\right)^{k}|f|\right)|Q| .
\end{aligned}
$$

Now we notice that since the cubes from $\tilde{\mathcal{S}}$ are dyadic, for every $l \in \mathbb{N}$,

$$
\begin{aligned}
\left(\sum_{P \in \tilde{\mathcal{S}}, P \subseteq Q} \eta_{P} \chi_{P}\right)^{l} & =\sum_{P_{1}, P_{2}, \ldots, P_{l} \subseteq Q, P_{i} \in \tilde{\mathcal{S}}} \eta_{P_{1}} \eta_{P_{2}} \ldots \eta_{P_{l}} \chi_{P_{1} \cap P_{2} \cap \ldots \cap P_{l}} \\
& \leq l ! \sum_{P_{l} \subseteq P_{l-1} \subseteq \ldots \subseteq P_{1} \subseteq Q, P_{i} \in \tilde{\mathcal{S}}} \eta_{P_{1}} \eta_{P_{2}} \ldots \eta_{P_{l}} \chi_{P_{l}} .
\end{aligned}
$$

Therefore,

$$
\int_{Q}|h|\left(\sum_{P \in \tilde{\mathcal{S}}, P \subseteq Q} \eta_{P} \chi_{P}\right)^{l} \leq l ! \sum_{P_{l} \subseteq P_{l-1} \subseteq \cdots \subseteq P_{1} \subseteq Q, P_{i} \in \tilde{\mathcal{S}}} \eta_{P_{1}} \eta_{P_{2}} \ldots \eta_{P_{l}}|h|_{P_{l}}\left|P_{l}\right| .
$$

Further,

$$
\begin{aligned}
& \sum_{P_{l} \subseteq P_{l-1} \subseteq \ldots \subseteq P_{1} \subseteq Q, P_{i} \in \tilde{\mathcal{S}}} \eta_{P_{1}} \eta_{P_{2}} \ldots \eta_{P_{t}}|h|_{P_{l}}\left|P_{l}\right| \\
= & \sum_{P_{l-1} \subseteq \ldots \subseteq P_{1} \subseteq Q, P_{i} \in \tilde{\mathcal{S}}} \eta_{P_{1}} \eta_{P_{2}} \ldots \eta_{P_{l-1}} \sum_{P_{l} \subseteq P_{l-1}, P_{l} \in \tilde{\mathcal{S}}}|h|_{P_{l}} \int_{P_{l}} \eta . \\
\leq & \sum_{P_{l-1} \subseteq \cdots \subseteq P_{1} \subseteq Q, P_{i} \in \tilde{\mathcal{S}}} \eta_{P_{1}} \eta_{L_{2}} \ldots \eta_{P_{l-1}} \int_{P_{l-1}} A_{\tilde{\mathcal{S}}}(|h|) \eta . \\
= & \sum_{P_{l-1} \subseteq \cdots \subseteq P_{1} \subseteq Q, P_{i} \in \tilde{\mathcal{S}}} \eta_{P_{1}} \eta_{L_{2}} \ldots \eta_{P_{l-1}}\left(A_{\tilde{\mathcal{S}}, \eta}|h|\right)_{P_{l-1}}\left|P_{l-1}\right|,
\end{aligned}
$$

where $A_{\tilde{\mathcal{S}}, \eta} h=A_{\tilde{\mathcal{S}}}(h) \eta$ and $A_{\tilde{\mathcal{S}}}(h)=\sum_{Q \in \tilde{\mathcal{S}}} h_{Q} \chi_{Q}$. Iterating this argument, we conclude that

$$
\int_{Q}|h|\left(\sum_{P \in \tilde{\mathcal{S}}, P \subseteq Q} \eta_{P} \chi_{P}\right)^{l} \lesssim \int_{Q} A_{\tilde{\mathcal{S}}, \eta}^{l}|h|,
$$

where $A_{\tilde{\mathcal{S}}, \eta}^{l}$ denotes the operator $A_{\tilde{\mathcal{S}}, \eta}$ iterated $l$ times. From this we obtain that the righthand side of 3.4 is controlled by

$$
\begin{aligned}
& c\|b\|_{B M O_{\eta}}^{m} \sum_{Q \in \tilde{\mathcal{S}}}\left(\frac{1}{|Q|} \int_{Q} A_{\tilde{\mathcal{S}}, \eta}^{k}(|f|)\right)\left(\frac{1}{|Q|} \int_{Q} A_{\tilde{\mathcal{S}}, \eta}^{m-k}(|g| \lambda)\right)|Q| \\
& =c\|b\|_{B M O_{\eta}}^{m} \int_{\mathbb{R}^{n}} A_{\tilde{\mathcal{S}}}\left(A_{\tilde{\mathcal{S}}, \eta}^{k}(|f|)\right) A_{\tilde{\mathcal{S}}, \eta}^{m-k}(|g| \lambda) .
\end{aligned}
$$


Using that the operator $A_{\tilde{\mathcal{S}}}$ is self-adjoint, we proceed as follows:

$$
\begin{aligned}
& \int_{\mathbb{R}^{n}} A_{\tilde{\mathcal{S}}}\left(A_{\tilde{\mathcal{S}}, \eta}^{k}(|f|)\right) A_{\tilde{\mathcal{S}}, \eta}^{m-k}(|g| \lambda)=\int_{\mathbb{R}^{n}} A_{\tilde{\mathcal{S}}}\left(A_{\tilde{\mathcal{S}}, \eta}^{k}(|f|)\right) A_{\tilde{\mathcal{S}}}\left(A_{\tilde{\mathcal{S}}, \eta}^{m-k-1}(|g| \lambda)\right) \eta \\
& =\int_{\mathbb{R}^{n}} A_{\tilde{\mathcal{S}}}\left(A_{\tilde{\mathcal{S}}}\left(A_{\tilde{\mathcal{S}}, \eta}^{k}(|f|)\right) \eta\right) A_{\tilde{\mathcal{S}}, \eta}^{m-k-1}(|g| \lambda)=\int_{\mathbb{R}^{n}} A_{\tilde{\mathcal{S}}}\left(A_{\tilde{\mathcal{S}}, \eta}^{k+1}(|f|)\right) A_{\tilde{\mathcal{S}}, \eta}^{m-k-1}(|g| \lambda) \\
& =\cdots=\int_{\mathbb{R}^{n}} A_{\tilde{\mathcal{S}}}\left(A_{\tilde{\mathcal{S}}, \eta}^{m}(|f|)\right)|g| \lambda .
\end{aligned}
$$

Combining the obtained estimates with $(3.3)$ yields

$$
\left\|A_{b}^{m, k} f\right\|_{L^{p}(\lambda)} \lesssim\|b\|_{B M O_{\eta}}^{m}\left\|A_{\tilde{\mathcal{S}}}\left(A_{\tilde{\mathcal{S}}, \eta}^{m}(|f|)\right)\right\|_{L^{p}(\lambda)} .
$$

Applying that $\left\|A_{\tilde{\mathcal{S}}}\right\|_{L^{p}(w)} \lesssim[w]_{A_{p}}^{\max }\left\{1, \frac{1}{p-1}\right\}$ (see, e.g., $|5|$ ), we obtain

$$
\begin{aligned}
\left\|A_{\tilde{\mathcal{S}}}\left(A_{\tilde{\mathcal{S}}, \eta}^{m}(|f|)\right)\right\|_{L^{p}(\lambda)} & \lesssim[\lambda]_{A_{p}}^{\max \left\{1, \frac{1}{p-1}\right\}}\left\|A_{\tilde{\mathcal{S}}, \eta}^{m}(|f|)\right\|_{L^{p}(\lambda)} \\
& =[\lambda]_{A_{p}}^{\max \left\{1, \frac{1}{p-1}\right\}}\left\|A_{\tilde{\mathcal{S}}}\left(A_{\tilde{\mathcal{S}}, \eta}^{m-1}(|f|)\right)\right\|_{L^{p}\left(\lambda \eta^{p}\right)} \\
& \lesssim\left([\lambda]_{A_{p}}\left[\lambda \eta^{p}\right]_{A_{p}}\right)^{\max \left\{1, \frac{1}{p-1}\right\}}\left\|A_{\tilde{\mathcal{S}}, \eta}^{m-1}(|f|)\right\|_{L^{p}\left(\lambda \eta^{p}\right)} \\
& \lesssim\left([\lambda]_{A_{p}}\left[\lambda \eta^{p}\right]_{A_{p}}\left[\lambda \eta^{2 p}\right]_{A_{p}} \ldots\left[\lambda \eta^{m p}\right]_{A_{p}}\right)^{\max \left\{1, \frac{1}{p-1}\right\}}\|f\|_{L^{p}\left(\lambda \eta^{m p}\right)} .
\end{aligned}
$$

Hence, setting $\eta=\nu^{1 / m}$, where $\nu=(\mu / \lambda)^{1 / p}$ and applying $(3.6)$, we obtain

$$
\left\|A_{b}^{m, k} f\right\|_{L^{p}(\lambda)} \lesssim\|b\|_{B M O_{\nu^{1 / m}}}^{m}\left([\lambda]_{A_{p}}[\mu]_{A_{p}} \prod_{i=1}^{m-1}\left[\lambda^{1-\frac{i}{m}} \mu^{\frac{i}{m}}\right]_{A_{p}}\right)^{\max \left\{1, \frac{1}{p-1}\right\}}\|f\|_{L^{p}(\mu)} .
$$

By Hölder's inequality,

$$
\prod_{i=1}^{m-1}\left[\lambda^{1-\frac{i}{m}} \mu^{\frac{i}{m}}\right]_{A_{p}} \leq \prod_{i=1}^{m-1}[\lambda]_{A_{p}}^{1-\frac{i}{m}}[\mu]_{A_{p}}^{\frac{i}{m}}=\left([\lambda]_{A_{p}}[\mu]_{A_{p}}\right)^{\frac{m-1}{2}}
$$

which, along with the previous estimate, yields

$$
\left\|A_{b}^{m, k} f\right\|_{L^{p}(\lambda)} \lesssim\|b\|_{B M O_{\nu^{1 / m}}}^{m}\left([\lambda]_{A_{p}}[\mu]_{A_{p}}\right)^{\frac{m+1}{2} \max \left\{1, \frac{1}{p-1}\right\}}\|f\|_{L^{p}(\mu)},
$$

and therefore the proof of part (i) is complete.

3.2. Proof of part (ii). Since $\mu, \lambda \in A_{p}$, by Hölder's inequality, it follows that $\nu^{1 / m} \in A_{2}$. Therefore, by Lemma 2.1, it suffices to show that there exists $c>0$ such that for all $Q$,

$$
\omega_{\frac{1}{2^{n+2}}}(b ; Q) \leq c\left(\nu^{1 / m}\right)_{Q} .
$$

The proof of (3.7) is based on the following auxiliary statement.

Proposition 3.1. There exist $0<\varepsilon_{0}, \xi_{0}<1$ and $k_{0}>1$ depending only on $\Omega$ and $n$ such that the following holds. For every cube $Q \subset \mathbb{R}^{n}$, there exist measurable sets $E \subset Q, F \subset k_{0} Q$ and $G \subset E \times F$ with $|G| \geq \xi_{0}|Q|^{2}$ such that

(i) $\omega_{\frac{1}{2^{n+2}}}(b ; Q) \leq|b(x)-b(y)|$ for all $(x, y) \in E \times F$;

(ii) $\Omega\left(\frac{x-y}{|x-y|}\right)$ and $b(x)-b(y)$ do not change sign in $E \times F$;

(iii) $\left|\Omega\left(\frac{x-y}{|x-y|}\right)\right| \geq \varepsilon_{0}$ for all $(x, y) \in G$. 
Let us show first how to prove (3.7) using this proposition. Combining properties (i) and (iii) yields

$$
\omega_{\frac{1}{2^{n+2}}}(b ; Q)^{m}|G| \leq \frac{1}{\varepsilon_{0}} \iint_{G}|b(x)-b(y)|^{m}\left|\Omega\left(\frac{x-y}{|x-y|}\right)\right| d x d y .
$$

From this, and using also that $|x-y| \leq \frac{k_{0}+1}{2} \operatorname{diam} Q$ for all $(x, y) \in G$, we obtain

$$
\omega_{\frac{1}{2^{n+2}}}(b ; Q)^{m}|G| \leq \frac{1}{\varepsilon_{0}}\left(\frac{k_{0}+1}{2} \sqrt{n}\right)^{n}|Q| \iint_{G}|b(x)-b(y)|^{m}\left|\Omega\left(\frac{x-y}{|x-y|}\right)\right| \frac{d x d y}{|x-y|^{n}} .
$$

By property (ii), $(b(x)-b(y))^{m} \Omega\left(\frac{x-y}{|x-y|}\right)$ does not change sign in $E \times F$. Hence, taking also into account that $|G| \geq \xi_{0}|Q|^{2}$, we obtain

$$
\begin{aligned}
\omega_{\frac{1}{2^{n+2}}}(b ; Q)^{m} & \leq \frac{1}{\varepsilon_{0} \xi_{0}}\left(\frac{k_{0}+1}{2} \sqrt{n}\right)^{n} \frac{1}{|Q|} \int_{E} \int_{F}|b(x)-b(y)|^{m}\left|\Omega\left(\frac{x-y}{|x-y|}\right)\right| \frac{d y d x}{|x-y|^{n}} \\
& =\frac{1}{\varepsilon_{0} \xi_{0}}\left(\frac{k_{0}+1}{2} \sqrt{n}\right)^{n} \frac{1}{|Q|} \int_{E}\left|\int_{F}(b(x)-b(y))^{m} \Omega\left(\frac{x-y}{|x-y|}\right) \frac{d y}{|x-y|^{n} \mid}\right| d x .
\end{aligned}
$$

Observing that $\left(T_{\Omega}\right)_{b}^{m}$ is represented as

$$
\left(T_{\Omega}\right)_{b}^{m} f(x)=\int_{\mathbb{R}^{n}}(b(x)-b(y))^{m} \Omega\left(\frac{x-y}{|x-y|}\right) f(y) \frac{d y}{|x-y|^{n}} \quad(x \notin \operatorname{supp} f),
$$

the latter estimate can be written as

$$
\omega_{\frac{1}{2^{n+2}}}(b ; Q)^{m} \leq \frac{c}{|Q|} \int_{E}\left|\left(T_{\Omega}\right)_{b}^{m}\left(\chi_{F}\right)\right| d x
$$

where $c$ depends only on $\Omega$ and $n$.

By Hölder's inequality,

$$
\frac{1}{|Q|} \int_{E}\left|\left(T_{\Omega}\right)_{b}^{m}\left(\chi_{F}\right)\right| d x \leq \frac{1}{|Q|}\left(\int_{E}\left|\left(T_{\Omega}\right)_{b}^{m}\left(\chi_{F}\right)\right|^{p} \lambda d x\right)^{1 / p}\left(\int_{Q} \lambda^{-\frac{1}{p-1}}\right)^{1 / p^{\prime}} .
$$

Using the main assumption on $T_{\Omega}$ along with the facts that $F \subset k_{0} Q$ and $\mu \in A_{p}$ and taking into account (2.1), we obtain

$$
\left(\int_{E}\left|\left(T_{\Omega}\right)_{b}^{m}\left(\chi_{F}\right)\right|^{p} \lambda d x\right)^{1 / p} \leq c \mu(F)^{1 / p} \leq c \mu(Q)^{1 / p},
$$

which, along with the previous estimate and (3.8), implies

$$
\omega_{1 / 2^{n+2}}(b ; Q)^{m} \leq c\left(\frac{1}{|Q|} \int_{Q} \mu\right)^{1 / p}\left(\frac{1}{|Q|} \int_{Q} \lambda^{-\frac{1}{p-1}}\right)^{1 / p^{\prime}} .
$$

By 2.3), $\frac{1}{|Q|} \int_{Q} \mu \leq c\left(\frac{1}{|Q|} \int_{Q} \mu^{1 / r}\right)^{r}$ for $r>1$. Further, by Hölder's inequality,

$$
\left(\frac{1}{|Q|} \int_{Q} \mu^{1 / r}\right)^{r} \leq\left(\frac{1}{|Q|} \int_{Q} \nu^{1 / m}\right)^{m p}\left(\frac{1}{|Q|} \int_{I} \lambda^{\frac{1}{r-m p}}\right)^{r-m p}
$$

Therefore, taking $r=m p+1$, we obtain

$$
\begin{aligned}
\omega_{1 / 2^{n+2}}(b ; Q)^{m} & \leq c\left(\frac{1}{|Q|} \int_{Q} \nu^{1 / m}\right)^{m}\left(\frac{1}{|Q|} \int_{Q} \lambda\right)^{1 / p}\left(\frac{1}{|Q|} \int_{Q} \lambda^{-\frac{1}{p-1}}\right)^{1 / p^{\prime}} \\
& \leq c\left(\frac{1}{|Q|} \int_{Q} \nu^{1 / m}\right)^{m}
\end{aligned}
$$


which proves (3.7).

Proof of Proposition 3.1. Let $\Sigma \subset S^{n-1}$ be an open set such that $\Omega$ does not change sign and not equivalent to zero there. Then there exists a point $\theta_{0} \in \Sigma$ of approximate continuity (see, e.g., [6, p. 46] for this notion) of $\Omega$ and such that $\left|\Omega\left(\theta_{0}\right)\right|=2 \epsilon_{0}$ for some $\epsilon_{0}>0$. By the definition of approximate continuity, for every $\varepsilon>0$,

$$
\lim _{\delta \rightarrow 0} \frac{\sigma\left\{\theta \in B\left(\theta_{0}, \delta\right) \cap S^{n-1}:\left|\Omega(\theta)-\Omega\left(\theta_{0}\right)\right|<\varepsilon\right\}}{\sigma\left\{B\left(\theta_{0}, \delta\right) \cap S^{n-1}\right\}}=1,
$$

where $B\left(\theta_{0}, \delta\right)$ denotes the open ball centered at $\theta_{0}$ of radius $\delta$, and $\sigma$ denotes the surface measure on $S^{n-1}$. Therefore, for every $0<\alpha<1$, one can find $\delta_{\alpha}>0$ such that

$$
B\left(\theta_{0}, \delta_{\alpha}\right) \cap S^{n-1} \subset \Sigma
$$

and

$$
\sigma\left\{\theta \in B\left(\theta_{0}, \delta_{\alpha}\right) \cap S^{n-1}:|\Omega(\theta)| \geq \varepsilon_{0}\right\} \geq(1-\alpha) \sigma\left\{B\left(\theta_{0}, \delta_{\alpha}\right) \cap S^{n-1}\right\} .
$$

Let $Q \subset \mathbb{R}^{n}$ be an arbitrary cube. Take the smallest $r>0$ such that $Q \subset B\left(x_{0}, r\right)$. Let $\theta \in B\left(\theta_{0}, \delta_{\alpha} / 2\right) \cap S^{n-1}$ and let $y=x_{0}+R \theta$, where $R>0$ will be chosen later. Our goal is to choose $R$ such that the estimate $\left|\frac{x-y}{|x-y|}-\theta_{0}\right|<\delta_{\alpha}$ will hold for all $x \in B\left(x_{0}, r\right)$.

Write $x \in B\left(x_{0}, r\right)$ as $x=x_{0}+\gamma \nu$, where $\nu \in S^{n-1}$ and $0<\gamma<r$. We have

$$
\frac{x-y}{|x-y|}=\theta+\frac{\gamma \nu-(R-|x-y|) \theta}{|x-y|} \text {. }
$$

Further,

$$
\begin{aligned}
\left|\frac{\gamma \nu-(R-|x-y|) \theta}{|x-y|}\right| & \leq \frac{\gamma}{|x-y|}+\frac{|R-| x-y||}{|x-y|} \\
& \leq \frac{2 \gamma}{|x-y|} \leq \frac{2 \gamma}{R-\gamma} \leq \frac{2 r}{R-r}
\end{aligned}
$$

For every $R \geq \frac{\left(4+\delta_{\alpha}\right) r}{\delta_{\alpha}}$ we have $\frac{2 r}{R-r} \leq \frac{\delta_{\alpha}}{2}$ and therefore,

$$
\left|\frac{x-y}{|x-y|}-\theta_{0}\right| \leq\left|\theta-\theta_{0}\right|+\frac{2 r}{R-r}<\delta_{\alpha} .
$$

Hence, setting

$$
\mathcal{F}_{\alpha}=\left\{x_{0}+R \theta: \theta \in B\left(\theta_{0}, \delta_{\alpha} / 2\right) \cap S^{n-1}, \frac{\left(4+\delta_{\alpha}\right) r}{\delta_{\alpha}} \leq R \leq \frac{\left(4+\delta_{\alpha}\right) 2 r}{\delta_{\alpha}}\right\}
$$

we obtain that

$$
\frac{x-y}{|x-y|} \in B\left(\theta_{0}, \delta_{\alpha}\right) \cap S^{n-1} \subset \Sigma \quad\left((x, y) \in Q \times \mathcal{F}_{\alpha}\right) .
$$

Also, it follows easily from the definition of $\mathcal{F}_{\alpha}$ that

$$
\mathcal{F}_{\alpha} \subset k\left(\delta_{\alpha}, n\right) Q \quad \text { and } \quad\left|\mathcal{F}_{\alpha}\right| \geq \rho_{n} \frac{|Q|}{\delta_{\alpha}} .
$$

By $3.10, \Omega\left(\frac{x-y}{|x-y|}\right)$ does not change sign on $Q \times \mathcal{F}_{\alpha}$. Let us show now that choosing $\alpha$ small enough, we obtain that $\left|\Omega\left(\frac{x-y}{|x-y|}\right)\right|<\varepsilon_{0}$ on a small subset of $Q \times \mathcal{F}_{\alpha}$. Set

$$
N=\left\{\theta \in B\left(\theta_{0}, \delta_{\alpha}\right) \cap S^{n-1}:|\Omega(\theta)|<\varepsilon_{0}\right\}
$$


and

$$
\mathcal{G}_{\alpha}=\left\{(x, y) \in Q \times \mathcal{F}_{\alpha}: \frac{x-y}{|x-y|} \in N\right\} .
$$

Let us estimate $\left|\mathcal{G}_{\alpha}\right|$. For $x \in Q$ denote

$$
\mathcal{G}_{\alpha}(x)=\left\{y \in \mathcal{F}_{\alpha}: \frac{x-y}{|x-y|} \in N\right\} .
$$

Notice that by (3.9),

$$
\sigma(N) \leq \alpha \sigma\left(B\left(\theta_{0}, \delta_{\alpha}\right) \cap S^{n-1}\right) \leq c_{n} \alpha \delta_{\alpha}^{n-1} .
$$

Next, for all $(x, y) \in Q \times \mathcal{F}_{\alpha}$ we have $|x-y| \leq c_{n}^{\prime} \frac{r}{\delta_{\alpha}}$, and hence,

$$
\left|\mathcal{G}_{\alpha}(x)\right| \leq\left|\left\{s \theta: 0 \leq s \leq c_{n}^{\prime} \frac{r}{\delta_{\alpha}}, \theta \in N\right\}\right| \leq c_{n}^{\prime \prime} \frac{|Q|}{\delta_{\alpha}^{n}} \sigma(N) \leq \beta_{n} \alpha \frac{|Q|}{\delta_{\alpha}} .
$$

Therefore,

$$
\left|\mathcal{G}_{\alpha}\right|=\int_{Q}\left|\mathcal{G}_{\alpha}(x)\right| d x \leq \beta_{n} \alpha \frac{|Q|^{2}}{\delta_{\alpha}} .
$$

Combining this with the second part of (3.11), we obtain that there exists $\alpha_{0}<1$ depending only on $n$ such that

$$
\left|\mathcal{G}_{\alpha_{0}}\right| \leq \frac{1}{2^{n+5}}\left|\mathcal{F}_{\alpha_{0}}\right||Q|
$$

By the definition of $\omega_{1 / 2^{n+2}}(b ; Q)$, there exists a subset $\mathcal{E} \subset Q$ with $|\mathcal{E}|=\frac{1}{2^{n+2}}|Q|$ such that for every $x \in \mathcal{E}$,

$$
\omega_{1 / 2^{n+2}}(b ; Q) \leq\left|b(x)-m_{b}\left(\mathcal{F}_{\alpha_{0}}\right)\right| .
$$

Next, there exist subsets $E \subset \mathcal{E}$ and $F \subset \mathcal{F}_{\alpha_{0}}$ such that $|E|=\frac{1}{2^{n+3}}|Q|$ and $|F|=\frac{1}{2}\left|\mathcal{F}_{\alpha_{0}}\right|$, and, moreover,

$$
\left|b(x)-m_{b}\left(\mathcal{F}_{\alpha_{0}}\right)\right| \leq|b(x)-b(y)|
$$

for all $x \in E, y \in F$ and $b(x)-b(y)$ does not change sign in $E \times F$. Indeed, take $E$ as a subset of either

$$
E_{1}=\left\{x \in \mathcal{E}: b(x) \geq m_{b}\left(\mathcal{F}_{\alpha_{0}}\right)\right\} \quad \text { or } \quad E_{2}=\left\{x \in \mathcal{E}: b(x) \leq m_{b}\left(\mathcal{F}_{\alpha_{0}}\right)\right\}
$$

with $\left|E_{i}\right| \geq \frac{1}{2}|\mathcal{E}|$, and the corresponding $F$ will be either $\left\{y \in \mathcal{F}_{\alpha}: b(y) \leq m_{b}\left(\mathcal{F}_{\alpha_{0}}\right)\right\}$ with $|F|=\frac{1}{2}\left|\mathcal{F}_{\alpha_{0}}\right|$ or its complement.

Combining 3.13 and 3.14 yields property (i) of Proposition 3.1. Also, since $\Omega\left(\frac{x-y}{|x-y|}\right)$ does not change sign on $Q \times \mathcal{F}_{\alpha_{0}}$, we have that property (ii) holds as well. Next, setting $G=(E \times F) \backslash \mathcal{G}_{\alpha_{0}}$, we obtain, by the second part of $(3.11)$ and $(3.12)$, that

$$
|G| \geq|E||F|-\left|\mathcal{G}_{\alpha_{0}}\right| \geq \frac{1}{2^{n+5}}\left|\mathcal{F}_{\alpha_{0}}\right||Q| \geq \nu_{0}|Q|^{2}
$$

where $\nu_{0}$ depends only on $\Omega$ and $n$, and, moreover, property (iii) follows from the definition of $\mathcal{G}_{\alpha_{0}}$. Finally, notice that by the first part of (3.11), $F \subset \mathcal{F}_{\alpha_{0}} \subset k_{0} Q$ with $k_{0}=k\left(\delta_{\alpha_{0}}, n\right)$. Therefore, Proposition 3.1 is completely proved. 


\section{REMARKS AND COMPLEMENTS}

Remark 4.1. The second part of Theorem 1.1 leaves an interesting question whether the assumption on $\Omega$ that it does not change sign on some open subset from $S^{n-1}$ can be further relaxed. In particular, one can ask whether this part holds for arbitrary measurable function $\Omega$, which is not equivalent to zero.

Remark 4.2. Similar to [4, 10, 21], Theorem 1.1 can be applied to provide a weak factorization result for Hardy spaces. For example, following Holmes, Lacey and Wick [10], one can characterize the weighted Hardy space $H^{1}(\nu)$ but in terms of a single singular integral, as this was done by Uchiyama [21]. The following proposition can be proved exactly as Corollary 1.4 in $[10]$.

Proposition 4.3. Under the hypotheses and notation of Theorem 1.1 and for the class of operators $T_{\Omega}$ described in Remark 1.2, we have that

$$
\|f\|_{H^{1}(\nu)} \simeq \inf \left\{\sum_{i=1}^{\infty}\left\|g_{i}\right\|_{L^{p^{\prime}\left(\lambda^{1-p^{\prime}}\right)}}\left\|h_{i}\right\|_{L^{p}(\mu)}: f=\sum_{i=1}^{\infty}\left(g_{i}\left(T_{\Omega}\right) h_{i}-h_{i}\left(T_{\Omega}\right)^{*} g_{i}\right)\right\} .
$$

Remark 4.4. Comparing both parts of Theorem 1.1, for the class of operators described in Remark 1.2 we have that the $L^{p}(\mu) \rightarrow L^{p}(\lambda)$ boundedness of $\left(T_{\Omega}\right)_{b}^{m}$ is equivalent to the restricted $L^{p}(\mu) \rightarrow L^{p}(\lambda)$ boundedness. It is interesting that $B M O_{\nu^{1 / m}}$ does not appear in this statement, though it plays the central role in the proof.

Remark 4.5. Theorem 1.1 answers the following question: what is the relation between the boundedness properties of commutators of different order? Again, let $T_{\Omega}$ be a singular integral as in Remark 1.2. Assume that $w \in A_{p}$. Then Theorem 1.1 implies immediately that for every fixed $k, m \in \mathbb{N}, k \neq m$,

$$
\left(T_{\Omega}\right)_{b}^{m}: L^{p}(w) \rightarrow L^{p}(w) \Leftrightarrow\left(T_{\Omega}\right)_{b}^{k}: L^{p}(w) \rightarrow L^{p}(w) .
$$

As in the previous remark, this implication is linked by $B M O$.

However, in the case of different weights, an analogue of (4.1) is not true in any direction, as the following example shows.

Example 4.6. Let $n=1$ and let $H$ be the Hilbert transform. Set $\mu=|x|^{1 / 2}$ and $\lambda=1$. Then we obviously have that $\mu, \lambda \in A_{2}$. Define $\nu=(\mu / \lambda)^{1 / 2}=|x|^{1 / 4}$ and let $b=\nu^{1 / 2}=|x|^{1 / 8}$. Then $b \in B M O_{\nu^{1 / 2}}$, since for every interval $I \subset \mathbb{R}$,

$$
\frac{1}{\nu^{1 / 2}(I)} \int_{I}\left|\nu^{1 / 2}-\left(\nu^{1 / 2}\right)_{I}\right| d x \leq 2 .
$$

Therefore, by Theorem 1.1, $H_{b}^{2}: L^{2}(\mu) \rightarrow L^{2}$. On the other hand, taking $I_{\varepsilon}=(0, \varepsilon)$ with $\varepsilon$ arbitrary small, we obtain

$$
\frac{1}{\nu\left(I_{\varepsilon}\right)} \int_{I_{\varepsilon}}\left|\nu^{1 / 2}-\left(\nu^{1 / 2}\right)_{I_{\varepsilon}}\right| d x=\frac{5}{4 \varepsilon^{5 / 4}} \int_{0}^{\varepsilon}\left|x^{1 / 8}-\frac{8}{9} \varepsilon^{1 / 8}\right| d x \geq \frac{c}{\varepsilon^{1 / 8}} .
$$

Therefore, $b \notin B M O_{\nu}$ and hence, by Bloom's theorem, $[b, H]: L^{2}(\mu) \not \rightarrow L^{2}$.

On the other hand, set $\mu=|x|^{-1 / 2}$ and $\lambda=1$. Then again $\mu, \lambda \in A_{2}$. Define $\nu=(\mu / \lambda)^{1 / 2}=$ $|x|^{-1 / 4}$ and let $b=\nu$. Then, arguing exactly as above, we obtain that $b \in B M O_{\nu}$ (and hence, $\left.[b, H]: L^{2}(\mu) \rightarrow L^{2}\right)$ and $b \notin B M O_{\nu^{1 / 2}}$. Therefore, by Theorem 1.1. $H_{b}^{2}: L^{2}(\mu) \nrightarrow L^{2}$.

Remark 4.7. Compare the condition $b \in B M O_{\nu \frac{1}{m}}$ with $b \in B M O \cap B M O_{\nu}$ from the works 11, 14. First, as we mentioned before, if $\mu, \lambda \in A_{p}$, then $\nu=\left(\frac{\mu}{\lambda}\right)^{\frac{1}{p}} \in A_{2}$. 
Lemma 4.8. Let $u \in A_{2}$ and $r>1$. Then

$$
B M O_{u} \cap B M O \subseteq B M O_{u}{ }^{\frac{1}{r}} .
$$

Furthermore, the embedding (4.2) is strict, in general. Namely, for every $r>1$, there exists a weight $u \in A_{2}$ and a function $b \in B M O_{u \frac{1}{r}} \backslash B M O$.

Proof. By 2.3,

$$
\begin{aligned}
\frac{1}{u^{\frac{1}{r}}(Q)} \int_{Q}\left|b(x)-b_{Q}\right| d x & \leq \frac{c}{u(Q)^{\frac{1}{r}}|Q|^{\frac{1}{r^{\prime}}}} \int_{Q}\left|b(x)-b_{Q}\right| d x \\
& =c\left(\frac{1}{u(Q)} \int_{Q}\left|b(x)-b_{Q}\right| d x\right)^{\frac{1}{r}}\left(\frac{1}{|Q|} \int_{Q}\left|b(x)-b_{Q}\right| d x\right)^{\frac{1}{r^{\prime}}},
\end{aligned}
$$

from which 4.2 readily follows.

To show the second part of the lemma, we use the same idea as in Example 4.6. Let $u(x)=|x|^{\alpha}, 0<\alpha<n$. Then $u \in A_{2}$. Let $b=u^{1 / r}=|x|^{\alpha / r}$. Then $b \in B M O_{u^{1 / r}}$. However, $b \notin B M O$, since it is clear that $b$ does not satisfy the John-Nirenberg inequality.

Take an integer $m \geq 2$. In accordance with Lemma 4.8, take $u \in A_{2}$ and $b \in B M O_{u} \frac{1}{m p} \backslash$ $B M O$. Then, setting $\mu=u$ and $\lambda=1$, by Theorem 1.1 we obtain that $T_{b}^{m}: L^{p}(u) \rightarrow L^{p}$ for every $p \geq 2$. This kind of estimates is not covered in [11] due to the fact that $b \notin B M O$.

Very recently we have learned that after finishing this paper some results concerning the necessity of $B M O$ for the endpoint estimate of commutators have been obtained. We remit the interested reader to [1] and [9] for those results. In an even more recent work [12], a more general version of the second part of Theorem 1.1 has been obtained, answering positively a question posed in Remark 4.1.

\section{ACKNOWLEDGEMENTS}

The first author was supported by ISF grant No. 447/16 and ERC Starting Grant No. 713927. The second was supported by CONICET PIP 11220130100329CO, Argentina. The third author was supported by the Basque Government through the BERC 2014-2017 program and by the Spanish Ministry of Economy and Competitiveness MINECO through BCAM Severo Ochoa excellence accreditation SEV-2013-0323 and also through the projects MTM2014-53850-P and MTM2012-30748.

\section{REFERENCES}

[1] Natalia Accomazzo. A characterization of BMO in terms of endpoint bounds for commutators of singular integrals. To appear in Israel J. Math. (Sept. 2017). arXiv: 1509.03769 [math.CA].

[2] Steven Bloom. A commutator theorem and weighted BMO. Trans. Amer. Math. Soc. 292.1 (1985), pp. 103-122. ISSN: 0002-9947.

[3] Daewon Chung, María Cristina Pereyra, and Carlos Pérez. Sharp bounds for general commutators on weighted Lebesgue spaces. Trans. Amer. Math. Soc. 364.3 (2012), pp. 1163-1177. ISSN: 0002-9947.

[4] Ronald R. Coifman, Richard Rochberg, and Guido Weiss. Factorization theorems for Hardy spaces in several variables. Ann. of Math. (2) 103.3 (1976), pp. 611-635. ISSN: 0003-486X.

[5] David Cruz-Uribe, José María Martell, and Carlos Pérez. Sharp weighted estimates for classical operators. Adv. Math. 229.1 (2012), pp. 408-441. ISSN: 0001-8708. 
[6] Lawrence C. Evans and Ronald F. Gariepy. Measure theory and fine properties of functions. Studies in Advanced Mathematics. CRC Press, Boca Raton, FL, 1992, pp. viii+268. ISBN: 0-8493-7157-0.

[7] José García-Cuerva, Eleonor Harboure, Carlos Segovia, and José Luis Torrea. Weighted norm inequalities for commutators of strongly singular integrals. Indiana Univ. Math. J. 40.4 (1991), pp. 1397-1420. ISSN: 0022-2518.

[8] Loukas Grafakos. Modern Fourier analysis. Third. Vol. 250. Graduate Texts in Mathematics. Springer, New York, 2014, pp. xvi+624. ISBN: 978-1-4939-1229-2; 978-1-49391230-8.

[9] Weichao Guo, Jiali Lian, and Huoxiong Wu. The unified theory for the necessity of bounded commutators and applications. ArXiv e-prints (Sept. 2017). arXiv: 1509.03769 [math.CA].

[10] Irina Holmes, Michael T. Lacey, and Brett D. Wick. Commutators in the two-weight setting. Math. Ann. 367.1-2 (2017), pp. 51-80. ISSN: 0025-5831.

[11] Irina Holmes and Brett D. Wick. Two Weight Inequalities for Iterated Commutators with Calderón-Zygmund Operators. ArXiv e-prints (Sept. 2015). arXiv: 1509.03769 [math.CA].

[12] T. P. Hytönen. The $L^{p}$-to- $L^{q}$ boundedness of commutators with applications to the Jacobian operator. ArXiv e-prints (Apr. 2018). arXiv: 1804.11167 [math.CA].

[13] Tuomas P. Hytönen. The $A_{2}$ theorem: remarks and complements. Harmonic analysis and partial differential equations. Vol. 612. Contemp. Math. Amer. Math. Soc., Providence, RI, 2014, pp. 91-106.

[14] Tuomas P. Hytönen. The Holmes-Wick theorem on two-weight bounds for higher order commutators revisited. Arch. Math. (Basel) 107.4 (2016), pp. 389-395. ISSN: 0003-889X.

[15] Gonzalo H. Ibañez-Firnkorn and Israel P. Rivera-Ríos. Sparse and weighted estimates for generalized Hörmander operators and commutators. ArXiv e-prints (Apr. 2017). arXiv: 1704.01018 [math.CA].

[16] Svante Janson. Mean oscillation and commutators of singular integral operators. Ark. Mat. 16.2 (1978), pp. 263-270. ISSN: 0004-2080.

[17] Fritz John. Quasi-isometric mappings. Seminari 1962/63 Anal. Alg. Geom. e Topol., vol. 2, Ist. Naz. Alta Mat. Ediz. Cremonese, Rome, 1965, pp. 462-473.

[18] Andrei K. Lerner. A pointwise estimate for the local sharp maximal function with applications to singular integrals. Bull. Lond. Math. Soc. 42.5 (2010), pp. 843-856. ISSN: 0024-6093.

[19] Andrei K. Lerner, Sheldy Ombrosi, and Israel P. Rivera-Ríos. On pointwise and weighted estimates for commutators of Calderón-Zygmund operators. Adv. Math. 319 (2017), pp. 153 -181. ISSN: 0001-8708.

[20] Jan-Olov Strömberg. Bounded mean oscillation with Orlicz norms and duality of Hardy spaces. Indiana Univ. Math. J. 28.3 (1979), pp. 511-544. ISSN: 0022-2518.

[21] Akihito Uchiyama. On the compactness of operators of Hankel type. Tôhoku Math. J. (2) 30.1 (1978), pp. 163-171. ISSN: 0040-8735. 
(A. K. Lerner) Department of Mathematics, Bar-Ilan University, 5290002 Ramat Gan, Israel Email address: lernera@math.biu.ac.il

(S. Ombrosi) Departamento de Matemática, Universidad Nacional del Sur, Bahía Blanca, 8000, Argentina

Email address: sombrosi@uns.edu.ar

(I. P. Rivera-Ríos) Department of Mathematics, University of the Basque Country and BCAM, Basque Center for Applied Mathematics, Bilbao, Spain

Email address: petnapet@gmail.com 